\title{
WHAT INFORMATION CAN BE EXTRACTED \\ FROM RADIO DATA ABOUT THE EXISTENCE OF SUPERMASSIVE BLACK HOLES?
}

\author{
L. M. OZERNOY \\ P. N. Lebedev Physical Institute, Academy of Sciences of U.S.S.R., Moscow, U.S.S.R.
}

The most convincing arguments to prove or disprove the idea that a supermassive accreting black hole serves as an energy source for quasars and quasar-like phenomena in galactic nuclei may be extracted from the variability of their radiation. There are at present numerous data on optical and radio variability of a number of objects.

As for optical data their statistical analysis applied to the quasar 3C 273 showed that the source of activity is not a cluster of independently and accidentally flaring objects like supernovae, but is some coherent body (Gudzenko et al., 1968, 1971). At present there are about ten quasars and active galactic nuclei demonstrating the quasi-periodic character of their optical variations (they are listed in Ozernoy, 1973). Such a behavior of luminosity supports the conclusion that the source of activity is a single body.

The next problem to be solved is whether the source of activity is a collapsed body or not. The discussion based mainly on optical data showed that the answer is apparently not (Ozernoy, 1974). The main reason is that the periods observed are much larger than may be expected for a supermassive black hole.

Now basing the data on the radio variability of quasars, I should like to suggest a quite different approach to the problem. The most popular explanation of the radio variability suggested first by Shklovskij and then developed by van der Laan and others is based on the model of an isotropically expanding plasma cloud which contains a 'frozen-in' magnetic field and relativistic electrons radiating by the synchrotron mechanism in the magnetic field which decreases adiabatically during the expansion. This model explains qualitatively rather well some observations, but being confronted with observational data by a quantitative manner encounters a number of difficulties. It turns out that the reason for these difficulties is that one assumes that the thermal plasma is responsible for the dynamics of the expansion. Meanwhile Ozernoy and Ulanovsky (1974) showed that two other kinds of models for variability are possible if the dynamics of the expansion is controlled by the magnetic field or relativistic particles, correspondingly. The best fit of observational data yields the model based on the assumption that the magnetic field controls the dynamics of the expansion. On being applied to some radio variable quasars, the model shows that at radius $R \sim 3 \times 10^{16} \mathrm{~cm}$ the magnetic field is as large as $10^{4}-10^{6} \mathrm{G}$, depending on the radial or dipole character of the field.

Such a large magnetic field, in principle, may be obtained from the matter accreting onto a supermassive black hole. However, in the course of an accretion any excess 
of the magnetic energy density, $H^{2} / 8 \pi$, over the matter density energy, $\varrho c^{2}$, is improbable. Meanwhile for the quasar 3C 279 the observations yield $H^{2} / 8 \pi \gg \varrho c^{2}$. If such an inequality is confirmed by further measurements of the radiovariability as a common case, it should be a new difficulty for the explanation of energy release by means of an accreting black hole.

The value of the magnetic field given above is just of the same order of value as expected from the theory of a magnetoid, i.e. rotating magnetized supermassive body (e.g. Ozernoy and Usov, 1971; 1973a, b). This is a new reason for the preference of a magnetoid as energy source for quasars and galactic nuclei. In principle, the most inner part of such a source may contain a collapsed region of much smaller mass. The above mentioned arguments suggest that the noncollapsed parent body is responsible for the observing manifestations of the quasar and galactic activity.

Note added in proof. Very probable existence of 'proton winds' from the nuclei of Seyfert galaxies offers a new possibility to obtain severe constraints on the collapsed mass as a source of activity (see L. M. Ozernoy, Astron. Tsirk. USSR, No. 804, 1 (1973)).

\section{References}

Gudzenko, L. I., Ozernoy, L. M., and Chertoprud, V. E.: 1968, Astron. Zh. 45, 492. Gudzenko, L. I., Ozernoy, L. M., and Chertoprud, V. E.: 1971, Astron. Zh. 48, 472. Ozernoy, L. M.: 1974, Proc. First Europ. Astron. Meeting, Vol. 3, Springer Verlag. Ozernoy, L. M. and Ulanovskij, L. E.: 1974, Astron. Zh. 51, 8.

Ozernoy, L. M. and Usov, V. V.: 1971, Astrophys. Space Sci. 13, 3.

Ozernoy, L. M. and Usov, V. V.: 1973a, Astrophys. Letters 13, 209.

Ozernoy, L. M. and Usov, V. V.: 1973b, Astrophys. Space Sci. 25, 149. 